\title{
Generalized Set Analysis of Sub-Saharan African Rhythm? Evaluating and Expanding the Theories of Willie Anku
}

Michael Scott Cuthbert

Massachusetts Institute of Technology, USA

\begin{abstract}
Willie Anku's rhythmic analyses relate the structure of drum patterns to recurring 12- or 16-beat rhythmic sets. Although Anku asserted that the distance between the start of these sets and a fixed time point was an important feature of a work, his rules for labelling these sets were not made explicit. This paper uses a computer simulation that trained on parts of Anku's analyses to predict the labels Anku used in other parts of his analyses. The simulation's rules are then extracted and presented to aid future analyses. The paper also expands Anku's concepts of pivot sets, prime forms of sets, and attack density.
\end{abstract}

\section{Introduction}

In a series of pamphlets and articles published from 1992 to 2000, the Ghanaian music theorist Willie Anku set forth theories of organization in African rhythm, particularly concerning the structure of recurring drum patterns which he termed "sets". Anku joined theorists, including Jeff Pressing (1983) and Jay Rahn (1987), in applying settheoretical analysis to the study of African music, adapting tools originally designed to describe and interpret pitch sets to what they saw as an isomorphic structure: beat classes in African drumming. Anku's theories have been well received and often cited, yet they have been sparsely applied by other researchers. There has been little published reflection on the implications of his analyses. That Anku himself has only employed his set analysis on two major works suggests that application of these theories could be a difficult and time-consuming labour.
This paper explores possible assumptions and methodologies which seem to be inherent in Anku's theories in order to begin to analyse his findings and consider their ramifications. Although Anku believes his theories operate on many repertories of sub-Saharan music, in this paper I will mostly evaluate Anku's theories in the context of the master drum line of Akan Adowa and Dagaaba Bawa dances.

\section{Anku's theory of regulative time points}

The most pervasive of Anku's theories has been that of the regulative time point (RTP). Anku uses RTP as a way of classifying individual lines by their rhythmic relationships to the timeline. Simply put, RTP shows on which pulse of a given line the strong pulse of the timeline falls.

Labelling the RTP of each line of Figure 1 does not present a problem because the example occurs in isolation. The first line of this eight-pulse passage is RTP 1 because the start of the line coincides with the strong pulse of the timeline, indicated with down arrows $(\downarrow)$. The second line is in RTP 5 because the strong pulse falls on the fifth pulse of the line. This reference point is not necessarily a sounded downbeat in Anku's view and in many instances would not be metrically accented; instead it is better conceived as structurally or perceptually strong (Anku, 1995, p. 177). Indeed it is the lack of acoustic accent in some genres that allows for listeners of different backgrounds and ethnicities to perceive different structural accents when hearing the same line.

Not all lines are so simple to label; Figure 2 shows some of the complexities of analysis. In this example, a new pattern begins eight beats before $\mathrm{A}$ and the strong 
beat of the timeline falls on the ninth beat of the line, so it is RTP 9. By B, though, the pattern has begun one pulse later, so we could label the second repetition as RTP 8 . We should note that from A on, RTP 2 also appears as a possibility.

Of course, knowledge of a particular musical style would be a tremendous aid to determining the conceptual strong beat, and thus the RTP of a passage - here, for instance, the Adowa verbal clue "Tつme kume menie" makes RTP 9 more logical than 2 for the phrase including the strong pulse A. However, Anku intended RTP and rhythmic sets to be tools for the analysis not just of a piece of music, with piece being construed broadly to include its context, but also as tools for the analysis of the piece as narrowly construed, that is of its transcription. This view is necessary to Anku's project. For it is only with this view of pieces that one can make broad claims about the wider structure of rhythm in an area as linguistically and ethnically diverse as Sub-Saharan Africa. In the absence of knowledge of verbal clues given by context, Anku's published writings do not tell us how we might decide between these two RTP interpretations. In general the writings have not explicitly given

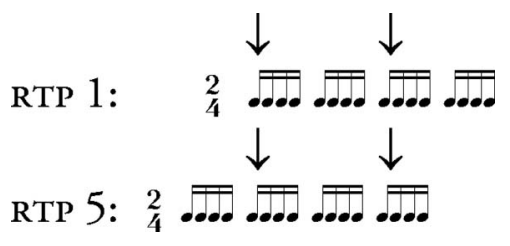

Fig. 1. RTP as a measure of the relationship between the perceived start of a set and the agreed upon felt strong pulse or downbeat of the work. principles for determining the RTP orientation of a span in the absence of particular cultural knowledge; the knowledge which Anku suggests is not generally necessary for set analysis. This is the point of tension in Anku's theories which gives them their greatest vitality. The theories involve a search not only for cross-cultural i.e. universal - analytical methods, but simultaneously for methods which place specific cultures' modes of perception at their centres.

Much of this paper's project is a search for such rules for deciding among conflicting possible analyses. I should emphasize though that the search is being conducted by an outsider to these musical forms - an outsider, not only in the sense of someone who did not grow up hearing in these ways, but as one who still does not know these perceptual forms for most of these repertories. This might serve me well at first in not being influenced by specific cultural norms in formulating the more general rules. But as the paper continues, I will increasingly emphasize the importance of turning to expert knowledge to explain why certain rules have explanatory force, what is the effect of certain parts of a work which are striking from an analytical point of view, and how refinements to a crude model can be implemented to better accord with performers' interpretations.

\section{Observations relating to RTP}

Turning to Anku's implied rules for determining what constitutes a rhythmic set, some observations were obvious and easy to describe. For example, the beginning of a rhythmic set is usually preceded by one or more rests: see Figure 3.

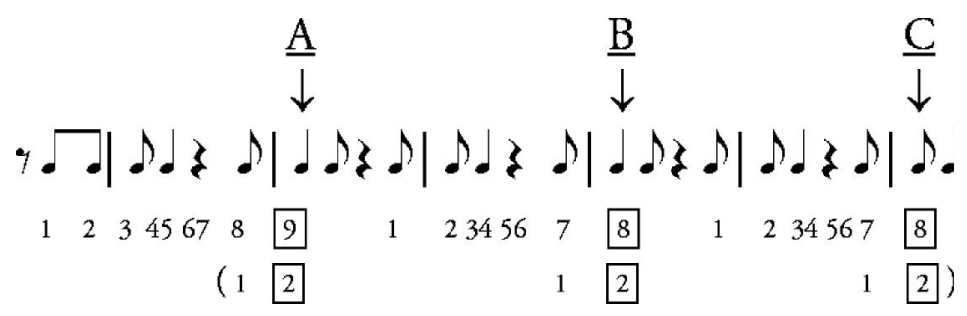

Fig. 2. The RTP of most passages with changing rhythms can be more difficult to determine, particularly for listeners of different ethnicities or audiences unfamiliar with other clues such as text-based themes or dance patterns (from Anku, 1997, pp. 234235 , bars $17-22$ ).

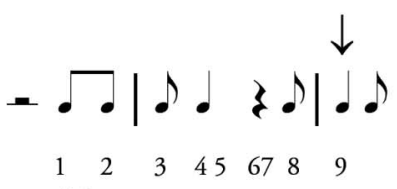

(a) RTP 9

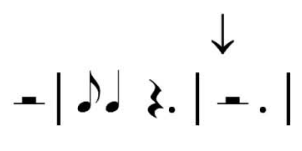

1234567

(b) RTP 7

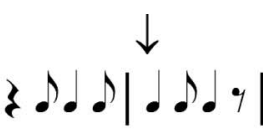

12345

(c) RTP 5

Fig. 3. The beginning of a set is usually preceded by one or (preferably) more rests (from Anku, 1992, (a) bars 5-7, (b) bars 57-59, (c) bars 170-71: the RTP labels are Anku's own). 
Note for instance in Figure 4, that a rest can also be hidden in a transcription by writing it as a quarter note, since quarter notes are merely orthographic conveniences in this style of transcription. A corollary of this observation is that the first beat of a group is nearly always attacked.

There are exceptions. Anku's RTP label for the 2nd donno part of the Adowa drum complex does not follow this rule, leading me to believe that the RTP of a line can be influenced by the RTP of a closely related line or instrument, in this case, the paired donno 1 line. See Figure 5. In Anku's version of the figure, he emphasizes the lines which do not attack the strong pulse of the timeline, such as the Apentemma and Donno 2, but does not specifically explain the significance of this lack of articulation.

Another principle implicit in Anku's theory is that of RTP inertia. In cases where it is difficult to determine the RTP, or two or more RTP seem possible, Anku is likely to

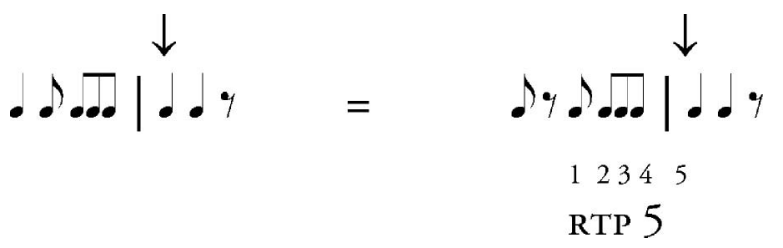

Fig. 4. A rest can be concealed by writing the previous note with durations longer than one pulse (from Anku, 1992, bars 94-95: again, the RTP label is Anku's). take into account the previous RTP and the RTP of the following section as well. In this same way, Western theorists have labelled the keys of ambiguous passages; for instance, we might know an E-major chord in an ambiguous context to be $\mathrm{V}$ and not $\mathrm{I}$ because of a previous strong A-major cadence. We see the principle of inertia in Anku's labelling of Bawa, where two identical, difficult to label passages are given the RTP of the preceding passages (see Figure 6).

In Figure 6, the small noteheads indicate "low velocity" strokes. The two eighth-note flams at the end of the second line can be seen as continuing the inertia of the previous RTP 7 theme. The light, dashed lines in the third system indicate subdivisions of what Anku considers a double-length set. In the 1993 edition of Bawa, the first quieter stroke was not transcribed, and thus this passage was considered as RTP 11 .

In order to apply these and other observations systematically, I decided to formalize rules about RTP and set grouping as pattern-matching instructions. These instructions matched segments of various works which I encoded. I should note that Anku himself used a computer program, drumbeats.c, to simplify working with set forms in his dissertation, so there is some analytical precedent for this method (Anku, 1988, pp. $272-274)$.

In order for a repertory to generate a meaningful RTP analysis it had to meet two basic criteria. First, it needed to have a meter, recurring timeline, or strong pulse whose

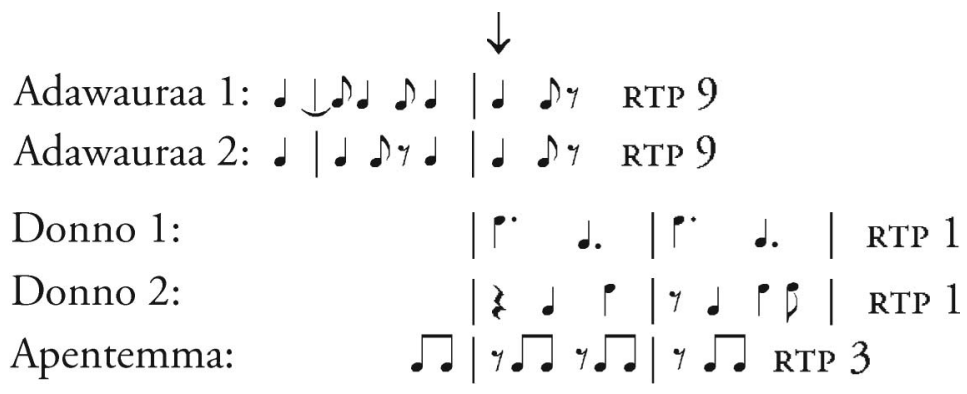

Fig. 5. The RTP of a line can be influenced by that of a closely related line. (Adapted from Anku, 1992, p. 6.)

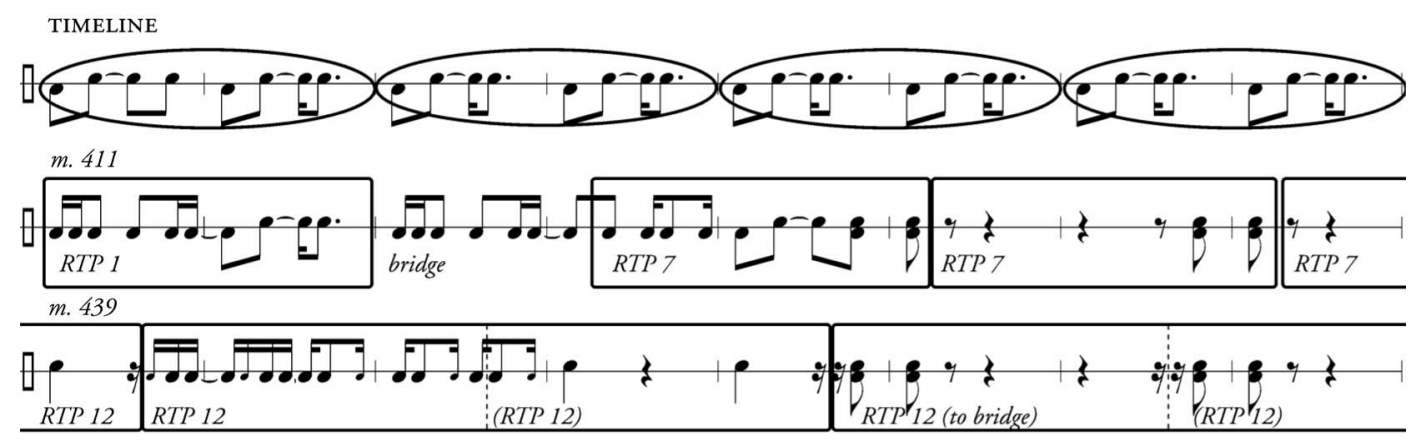

Fig. 6. Inertia as an important factor in determining RTP. (Adapted from Anku, 1993, revised version 2002.) 
structural referent was agreed upon by the players. This excluded from analysis non-dance repertories based strongly on speech rhythms, such as certain talking drum repertories. Secondly, to produce interesting results the orientation of a line (with respect to the strong beat) must change within the work. The support drum parts in Figure 5, for instance, usually do not shift with respect to the timeline. Similarly, many songs are in a single RTP throughout and would not be of interest to computeraided analysis. (The study of RTP frequency within a repertory or group of repertories within a region might form an interesting study. However, this paper, like those of Anku, examines RTP use only within an individual piece.) To keep this study focused but give some depth, I have chosen to focus on a single repertory, that of the atumpan lines of Adowa.

I developed twenty-six rules for labelling the RTP of sets. In keeping with Anku's desire to have RTP be generally applicable to sub-Saharan African music, only four of these rules were made specific to Adowa - these rules identify the nine-beat 'idiomatic bridges' which are transition points between large sections of a dance. Each rule has an attached numeric score showing how useful the particular rule is for labelling the set as a particular RTP, and the scores from the various rules are added to show how strongly a certain RTP might be felt. Readers might note that this approach of using rules and scorefiles is similar to the approach of many popular spam filters. (An important distinction is that there are 12 or 16 possible outputs rather than just the two, spam or not spam.) Although there are many ways to have the rules and the scores generated automatically, via Bayesian classifiers or neural nets, for instance, I chose to write the rules and tweak the scores by hand. I hoped that by exerting some personal control over the analytical process, I might avoid Meki Nzewi's wellfounded criticism of "human scientific wizards who abdicate their senses to the wonder machines, and, thereby, miss simple, common sense and natural solutions to natural human problems" (Nzewi, 1997, p. 23) and also to produce concepts more easily translatable to future, non-computer-based analyses. In Figure 7, I give

Example concept 1: A 12-beat pattern which is identical to the following 12 beats is roughly twice as strong an indicator of RTP as a 12-beat pattern which has only one difference from the following 12 beats.

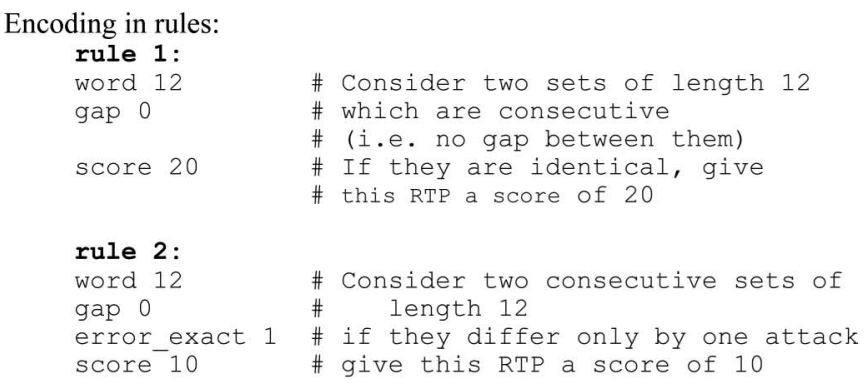

Example concept 2: Inertia. When a 6-beat pattern matches a 6-beat pattern 6 beats later, we should consider the current RTP more likely to be the RTP for the next pattern, and to a lesser extent for the pattern after that.

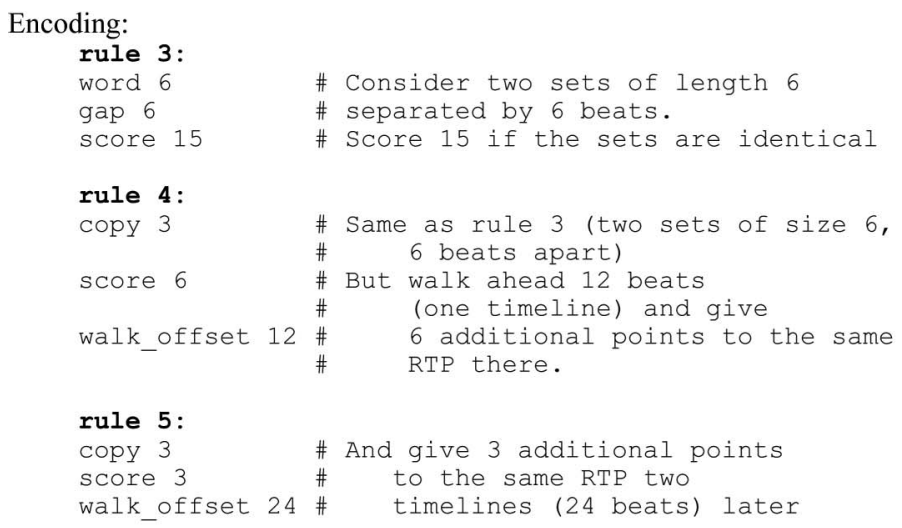

Other possible modifications were:

rfb attack: require the first beat of this RTP to be attacked

rp_silence: require the beat preceding the first beat to be silent tone_insensitive: when comparing two sets, ignore differences in pitch

Fig. 7. Two concepts and their encodings in five rules. 
two concepts in detail and five rules needed to implement them. (The complete scorefile and the program which evaluates the scorefile can be found online along with other materials at $<$ http: $/ /$ myke.trecento.com/anku/ $>$.) I will present an example of a place outside the training data where the program worked well. The following passage is from the beginning of an Adowa dance which Anku presents in a stylized performance. The transcription is Figure 8.

Anku labelled the passage as in Figure 9, while the automatic labelling program that I wrote produced the results in Figure 10.

For the passages marked "bridge", specific information about Adowa was used. The rest of the RTP were labelled only with the knowledge that the piece used twelve beat sets. Note that for the passage from bars $21-$ 22 (set 9), the highest scoring RTP was RTP 2, but it did not
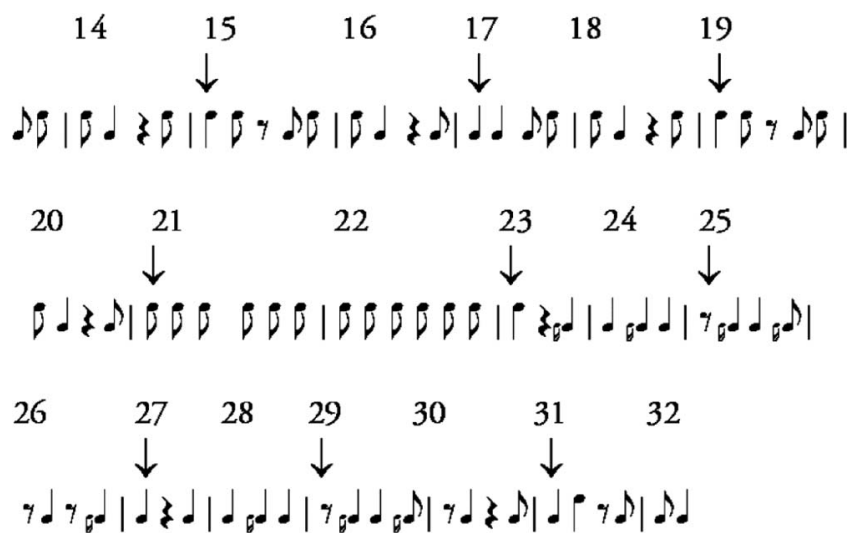

Fig. 8. Unlabelled transcription from Adowa: downward and upward stemmed notes are low and high attacks, respectively. ;. indicates a flam. score high enough to be considered a "strongly heard RTP". In the preceding passage, three different RTP were considered strongly emphasized, 2, 9 and 11, but 9 was considered the most strongly emphasized of the trio. I consider a metric of the amount of ambiguity in the line as important a part of the analysis as the RTP output itself.

The program labelled the shift from RTP 9 to 2 at the same place as Anku but went further to say that RTP 2 was much less strongly felt than the preceding RTP 9. In all, the program was able to label correctly $\sim 60 \%$ of Anku's RTP groups beyond the ones I trained it on (where it did nearly perfectly as it should have). $60 \%$ can be usable, but is not extraordinary. But I am intrigued that when RTP orientations considered by the program to be second or third choices were added, the percentage jumped to $\sim 75 \%$. The $15 \%$ of passages which second and third choices picked up will be the most interesting to pursue further; it may well be that these are the passages where knowledge of a particular style is necessary to override general rules. The question of whether further improvements can be made in a generalized manner - that is, without recourse to specific linguistic or ethnic perceptions - remains unresolved. But by making us attentive to particular passages, I hope this method of labelling can focus our future analytical and ethnographic researches.

\section{Expansions}

The framework which Anku has created can be expanded in several ways. In the examples I have used so far, the lengths of the master drum lines' sets have been either the same or (in Figure 6, line 3) twice as long as that of the timeline. In sets whose lengths are not multiples of

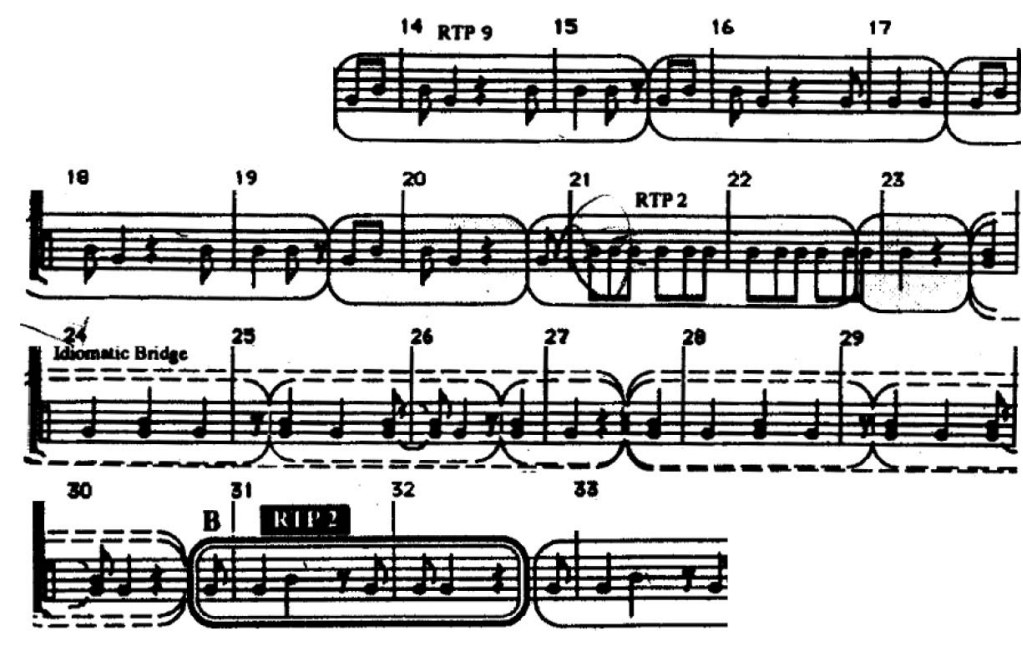

Fig. 9. Anku's transcription and analysis of the same passage (Anku, 1992, pp. 10-11). Anku transcribes the flam with both high and low noteheads. I have erased the bell line from this transcription. 


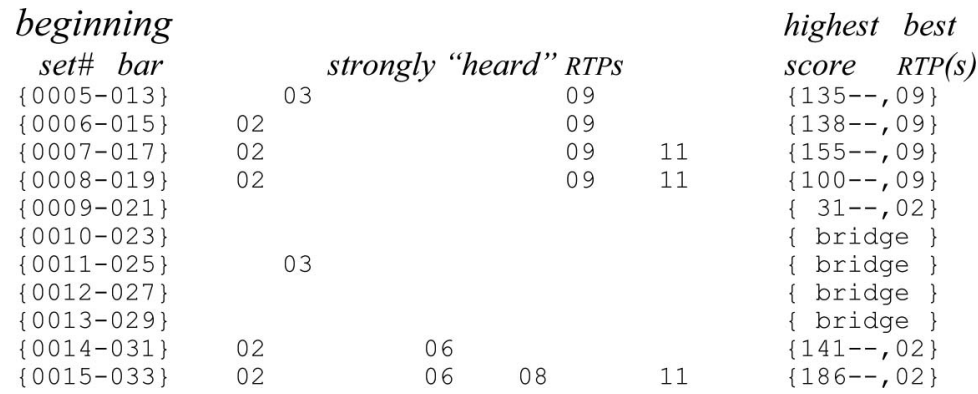

Fig. 10. Output from program which performed automatic set labelling.

the length of the time line, such as $3: 4$ or $3: 2$, the RTP of the passage does not remain constant even if the drum line remains constant, such as happens often in the Bawa repertory (Figure 11).

Shifts in RTP are supposed to signal moments of change in a work, so the frequent RTP shifts in Figure 11 seem to go against this model. A notation should be devised which allows sections such as this to remain in a single RTP throughout the passage while acknowledging that the start of the set was shifting with respect to the start of the timeline. In the 2002 version, the only label given is RTP 9. I believe Anku intends for the passage to be heard as constant, which should entail a constant RTP. A notation could be devised to show that although the set begins on other points in the timeline, it is essentially constant on a larger scale. One such notation might be RTP $9^{0 / 2}$, RTP $9^{1 / 2}$ which would show in the second case that the passage is essentially the first repetition of a RTP 9 passage which needs two repetitions in order to remain in a constant position with respect to the strong pulse of the bell pattern. Anku also allows for an interpretation with sets of length twelve (rather than eight or sixteen). In that case, rather than hearing successive sets in RTP 9, 13, 1 and 5, my proposed notation would keep the RTP constant at 9 and read RTP $9^{0 / 4}, 9^{1 / 4}, 9^{2 / 4}, 9^{3 / 4}$.

Another concept which could be expanded is Anku's use of 'pivot sets' which function both in a prior and upcoming RTP orientation in an analogous way to how pivot chords function to move from one key to another. Unlike pivot chords, the pivot set is usually identical to the main material of the preceding section; again, I draw my example, Figure 12, from the opening of Anku's Adowa transcription.

The ellipses show consecutive RTP 9 sections while the rectangle encompasses an RTP 2 set. The five pulses which fall in both shapes form Anku's pivot set. Since my analytical method gives a score to each RTP, it might be possible to show some pivots as stronger or smoother than others (in the same way as some chords are stronger or smoother pivots in tonal music) and it might also be possible to show larger sections which can be heard simultaneously in two or more RTP while gradually

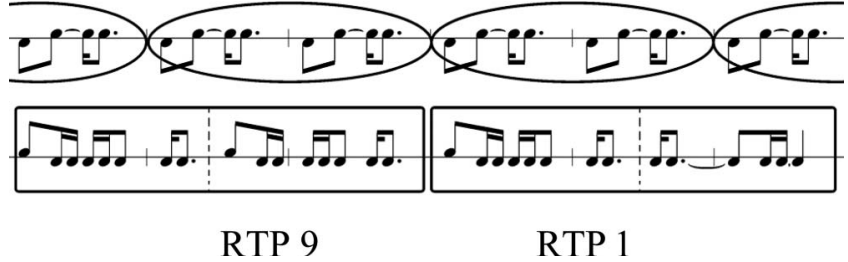

Fig. 11. Sets whose lengths are 3/4 times the length of the 16pulse timeline: interpretation from Anku (1993), transcription is adapted from the slightly revised version in 2002, bars 22-27. The recurring bell pattern is the first line (enclosed in ovals).

slipping from one set to another. However, David Locke's (1987, pp. 16-31) discussion of the "aural illusions" of polymeter and temporary cross-relations, while not discussing precisely this phenomenon, should be referenced here if only as a caveat. Locke states that although Africans do occasionally perceive a shift in beat orientation or even meter, this type of observation is made much more commonly by Westerners. Therefore, it is vital that this extension of Anku's theory in particular be reinforced by interview and testimony of expert performers.

\section{Prime forms}

For the final section of this paper, I wish to consider another analytical tool which Anku adopted from pitch set theory. In two recent articles, Anku has labelled what he calls the prime forms of various sets taken from African repertories. These prime forms are for rhythm the rough equivalent to Forte's (1973) normal forms, or more closely, Solomon's $(1988,2005)$ prime forms, that is the rotation of the beat class where the smallest intervals are at the beginning. Unlike Forte and Solomon, Anku does not require that the distance from the first to the last element of the set be minimized and begins instead by minimizing the distance between element 1 and 2, 2 and 3, etc. By this definition, a C-major chord would be rotated E-G-C ( $\langle 470\rangle$ or [038]) instead of C-E-G ([047]). 
The forms are then written as interval patterns, i.e. with the distance from the previous element noted rather than the distance from the start, and Anku also includes the interval to the start of the next set. That is, for the set $\left.\left\langle \_. . .\right\rangle\right\rangle$he would rotate it to [^....] and write [1122] where the last " 2 " shows the distance to the beginning of the next set. Anku, following Pressing and Rahn, shows how certain beat sets appear in different rotations among different cultures. The most important and famous of these is prime form [1221222], which he asserts is perceived in Ewe areas as rotation $\langle 2212221\rangle$, and by Yoruba as $\langle 2212212\rangle$, and so on. (This prime form is famous for its isomorphism to the diatonic scale whose notes are in major $\langle 2212221\rangle$ half steps apart and in natural minor $\langle 2122212\rangle$.)

Although Anku has asserted the power of prime forms for understanding rhythmic change within a work, he has not, however, labelled the prime forms of any work with more than nine different sets. Again with a computer, I labelled the sets in four large Adowa dances, of approximately fifty different sets each. A small, but not inconsequential, number of sets with the same prime form but different rotations appear in the same piece. Some beat-class sets such as [22224] are not surprising to find in several rotations. This is a common form of the idiomatic bridge, a nine or ten beat set which moves the line from one RTP to another. Multiple rotations of some other prime forms exist, as Figures 13 and 14 show.

Figure 14 shows three variants of beat class set [11415]. In all cases, the pattern was analysed by Anku as the complement of some preceding (often distant) incomplete set. It may be that the angular nature of [11415], with its fast notes interspersed by long rests, makes it particularly apt at completing prior incomplete sets. The third form of [11415] is peculiar from a set- theoretical standpoint. The perceived starting point of the set is not attacked. Although these forms are important in Anku's labels of RTP, and are well known in West African dances (Locke, 1982), they have not often been studied by set class theorists. For instance, they are omitted from discussion in Toussaint (2003). But they produce strikingly different effects than any of the standard rotations. The Highlife timeline $\langle\{\% \%\rangle$, for instance, is a rhythm which cannot be represented in any of the conventional rotational forms. (As this paper neared press, Agawu's article (2006) appeared including a significant discussion of rotation in this line. To account for such a line theoretically, he redefines rotation so that it no longer refers to the moving of onsets (p. 14) but instead to the moving of unnotated density referents (pp. 25-26), a theoretical construct he had previously attacked (p. 22).)

Empty-attack sets are isomorphic to pitch-class sets whose principal notes are not parts of the sets themselves. Consider for instance the possibility of a white-key scale in a rotation which begins on the absent note $\mathrm{C} \sharp$. Omitted notes, particularly roots, are common to tonal theory (consider the Riemannian $\mathrm{V}_{\mathrm{o}}{ }^{9}$ terminology), but have not played a major role in set theory. Thus, in the category of absent tonic sets, beat class theory of African music can give back to pitch set theory a large repertory of works of particular interest.

\section{Attack density}

One reason Anku began working with sets and prime forms was his a belief that patterns in the number of attacks in a set showed patterns in the intensity of the piece. However as Figures 15 and 16 show, what might look like a coherent bell-shaped pattern of intensity followed by alternation

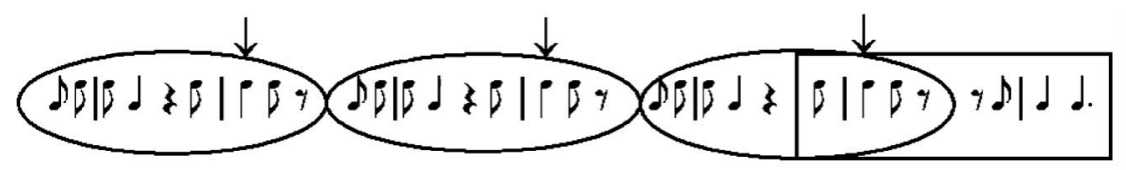

Fig. 12. Pivot sets: adapted from Anku (1995, pp. 179-180).

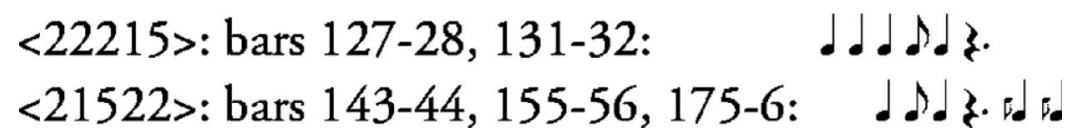

Fig. 13. Two forms of beat class with Anku prime form [15222]: Bar numbers refer to Anku (1992).

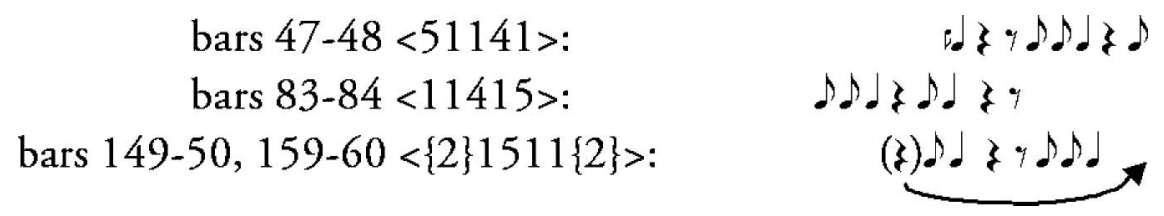

Fig. 14. Three forms of beat class [11415], the third beginning with an empty attack: bar numbers refer to Anku (1992). 


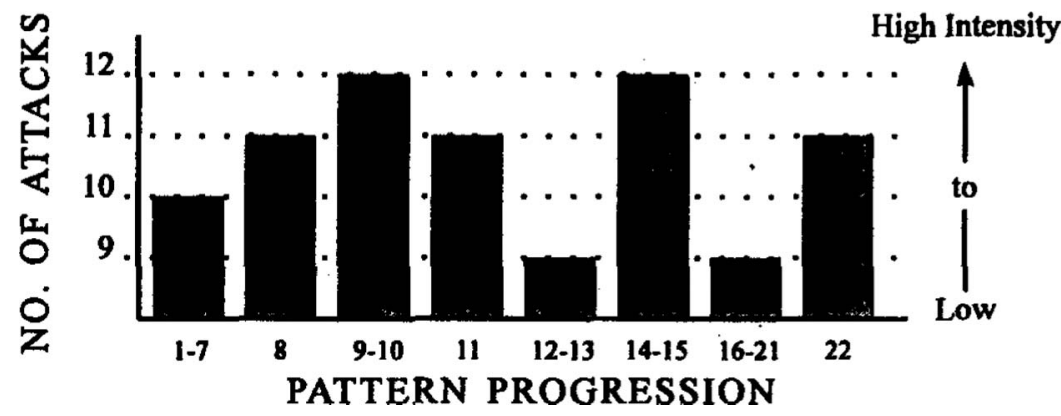

Fig. 15. Attack density in a performance of Samba (Anku's graph) (from Anku, 1988, p. 287 and Anku, 1995, p. 195).

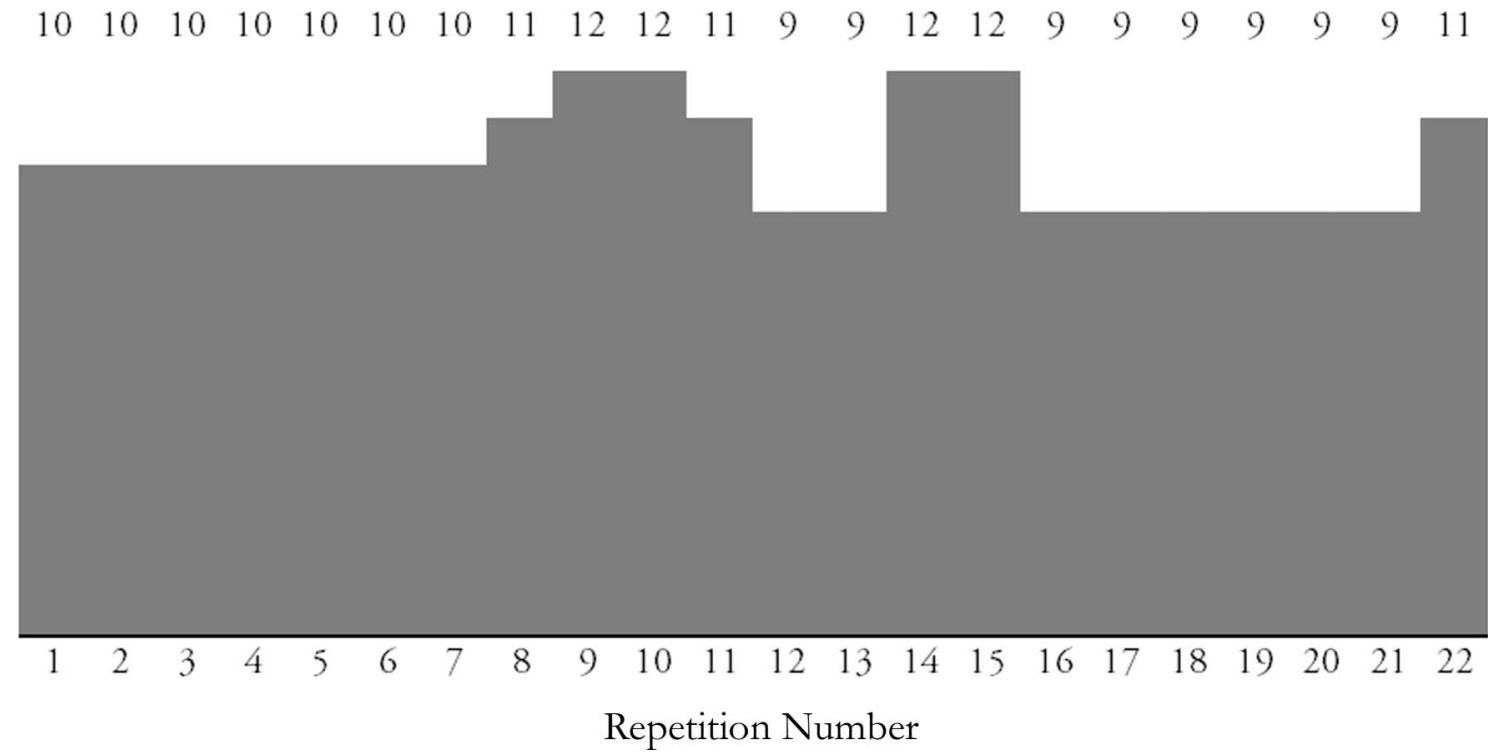

Fig. 16. Another view of attack density in the same performance of Samba.

between intense and weak sets is more likely the result of a distorted scale Anku employs for his graph.

I applied this method of attack density analysis to five larger works across three genres and found no coherent large-scale patterning in the music, except perhaps a flatness of attack density in certain works like the Samba (Figure 16). In my graph successive identical sets are not joined, so the horizontal scale represents constant time. In all, only four of the sixteen possible densities of attack are used, giving the impression of flatness of attack density rather than instability.

\section{Conclusions}

The theories of Willie Anku offer fresh attempts at providing analytical frameworks for rhythm across repertories in sub-Saharan Africa. Anku's grouping of the rhythms and tones of a line into sets provides a way both of considering the bar to bar (or timeline to timeline) structure of the work as well as the larger-scale form of the dance. It is my intention that by presenting rules and more encoded works to be analysed, Anku's theories might be more easily applied. But this result is not a goal in itself, but a means to better answer the questions which are so important to the study of a collection of music or a music culture. The questions here might include, are there places in pieces where a player with a changing RTP, such as the master drummer, feels that his part is synchronized with, or feels tension with, one or another of the support drum lines? Or vice versa for a support drum player? If so, is there a pattern to the RTP relationships between the two parts which coincides with these feelings? How do analyses of repertories which have verbal cues, such as Adowa, differ from those such as Bawa which do not? Is rotational equivalence of sets perceptible or can it be trained to be? (and if not, should this dissuade theorists from its use?) These are to me some of the most important questions, and while this paper can help them be formulated more precisely, no amount of scorefiles or encoded transcriptions will answer them. The crucial role of ethnic perception and ethnographic interview persists for even the most theoretical work on African rhythm. 


\section{Acknowledgements}

I wish to thank David Kaminsky, Meki Nzewi, and two anonymous readers for helpful comments on this paper. Particular thanks go to Kofi Agawu for the initial motivation to write and many discussions about this paper over the years. I likewise owe a tremendous debt of gratitude to Willie Anku for his encouragement of and interest in this work.

\section{References}

Agawu, K. (2006). Structural analysis or cultural analysis? Competing perspectives on the "standard pattern" of West African rhythm. Journal of the American Musicological Society, 59(1), 1-46.

Anku, W. (1988). Procedures in African drumming: A study of Akan/Ewe traditions and African drumming in Pittsburgh. PhD thesis, University of Pittsburgh, USA.

Anku, W. (1992). Structural Set Analysis of African Music 1: Adowa. Legon, Ghana: Soundstage Production. Revised 2002.

Anku, W. (1993). Structural Set Analysis of African Music 2: Bawa. Legon, Ghana: Soundstage Production. Revised 2002.

Anku, W. (1995). Towards a cross-cultural theory of rhythm in African drumming. In C.T. Kimberlin \& A. Euba (Eds.), Intercultural music, volume 1. pp. 167-202. Bayreuth: E. Breitinger.

Anku, W. (1997). Principles of rhythm integration in African drumming. Black Music Research Journal, $17(2), 211-238$.
Anku, W. (2000). Circles and time: A theory of structural organization of rhythm in African music. Music Theory Online, 6(1). Available online at: $<\mathrm{http}: / /$ boethius.music. ucsb.edu/mto/issues $/ \mathrm{mto} .00 .6 .1 / \mathrm{mto} .00 .6 .1$.anku.html $>$

Forte, A. (1973). The structure of atonal music. New Haven: Yale University Press.

Locke, D. (1982). Principles of offbeat timing and crossrhythm in Southern Eve dance drumming. Ethnomusicology, 26(2), 217-246.

Locke, D. (1987). Drum Gahu: A systematic method for an African percussion piece. Crown Point, Indiana: White Cliffs Media Company.

Nzewi, M. (1997). African music: Theoretical content and creative continuum: The culture-exponent's definitions. Olderhausen: Institut für Didaktik popularer Musik.

Pressing, J. (1983). Rhythmic design in the support drums of Agbadza. African Music, 61(1), 4-15.

Rahn, J. (1987). Asymmetrical ostinatos in sub-Saharan music: time, pitch, and cycles reconsidered. In Theory Only, 9(7), 23-37.

Solomon, L. (1998; rev. 2005). Why is the prime different from the Forte prime? Available online at: <http:/ solomonsmusic.net/setheory.htm >

Toussaint, G. (2003). Classification and phylogenetic analysis of African ternary rhythm timelines. In Proceedings of BRIDGES: Mathematical Connections in Art, Music, and Science, University of Granada, Granada, Spain, 23-26 July, pp. 25-36. Available online in an extended version at: <http://cgm.cs.mcgill.ca/ $\sim$ godfried/publications/ternary.pdf $>$ 